\title{
Difficulties in Diagnosis of Sialolithiasis: A Case Series
}

\author{
Svitlana Veniaminivna Kolomiiets, Kristina Oleksandrivna Udaltsova, \\ Tetiana Andriivna Khmil, Alina Mykolaiivna Yelinska, \\ Olena Anatoliivna Pisarenko and Viktoriya Ihorivna Shynkevych
}

\author{
Department of Postgraduate Education for Dentists, Higher State Educational \\ Establishment of Ukraine "Ukrainian Medical Stomatological Academy", \\ Poltava, Ukraine
}

Received 13 April, 2017/Accepted for publication 8 July, 2017

\begin{abstract}
Sialolithiasis is one of the most common and extensively obstructive disorders of the major salivary glands. Here, we report 3 cases of sialolithiasis in the submandibular salivary gland showing symptomatic similarities to other dental and non-dental disorders of the maxillofacial area. How the various clinical features of this condition and findings on 3D-CT may lead to a misdiagnosis are also discussed. In the first case, that of a 45-year-old woman, a final diagnosis of a non-radiopaque submandibular sialolith allowed the initially indicated surgical extraction of a malerupted and semi-impacted right mandibular 3rd molar to be abandoned. In the second case, that of a 57-year-old woman, radiographic findings had previously led to a diagnosis of ameloblastoma, which had masked the presence of sialolithiasis for at least 9 years, despite the radiopacity of the sialolith. Meanwhile, exacerbation of sialolithiasis was mistaken for lymphadenitis. In the third case, that of a 40-year-old woman, sialolithiasis was diagnosed in a timely manner, despite the fact that the dentists' attention had initially been focused on odontopathological symptoms. One feature of the present report is the concurrence of dental and non-dental pathologies affecting the same sextant as the sialolithiasis. Despite recent advances in imaging technology and diagnostics, cases of sialolithiasis being misdiagnosed continue to occur in clinical practice.
\end{abstract}

Key words: Diagnosis — Sialolithiasis — Submandibular salivary gland

\section{Introduction}

Sialolithiasis is one of the most prevalent and extensively obstructive disorders of the major salivary glands. In one recent study, it was estimated that the annual symptomatic incidence of sialoliths was 1 per 10,000 and 1 per 20,000 (individuals) ${ }^{8}$. The incidence rate of sialolithiasis has been reported to be between 7.27 and 14.10 per one hundred thousand (person-years), with sex showing no significant effect ${ }^{10)}$.

Most sialoliths develop in the submandibular salivary gland or its ducts (80 to $92 \%)$ and 
can cause acute and chronic inflammation ${ }^{7)}$. The predominant prevalence of sialoliths in the submandibular salivary glands is due to various anatomical and chemical factors, such as differences in the composition of the saliva.

Patients most often present with a tingling sensation and swelling of the glands after meals. The course of the disease is characterized by relapse and remission until final definitive treatment is required, usually in the form of a surgical intervention.

Despite the fact that the patient will generally require hospitalization for such treatment, the diagnosis itself is usually made by a dentist working in a private or municipal setting depending on the type of facilities available in the post-Soviet area in which they live.

A number of tools and research methods are commonly used to diagnose sialolithiasis. Both panoramic and occlusal radiographic techniques have been demonstrated to display satisfactory diagnostic screening performance $^{6)}$. Approximately $20 \%$ of sialoliths are poorly calcified, and therefore not visible on 2D radiographs ${ }^{5}$. Moreover, the deposition of a radiopaque sialolith on the body of the mandible may render the former barely visible radiographically.

High-frequency ultrasound examination (US) has been reported to be effective in confirming a diagnosis of sialolithiasis ${ }^{1)}$. Reconstructed conventional CT and volume rendering or 3D computed radiography (3D-CT) are used for imaging of the structures of the skull. These modalities offer the advantage of only a relatively low dose of radiation-absorbance $(50 \mathrm{mSv})$, which is contraindicated in pregnant patients only. The literature concludes that the diagnostic criteria of sialolithiasis are fairly well-known, and that this condition is commonly encountered in a clinical setting. However, despite the availability of a range of imaging techniques, the diagnosis of sialolithiasis may sometimes remain difficult ${ }^{2}$.

Here, we report 3 cases of sialolithiasis in the submandibular salivary glands accompanied by symptoms of other dental and nondental disorders of the maxillofacial area.
How the various clinical features of this condition and findings on 3D-CT may lead to a misdiagnosis are also discussed. The authors obtained written informed consent from the patients concerned to present materials from their case histories in this paper in compliance with the ethical approval protocol of this institute.

\section{Case Presentations}

\section{Case no.1}

This patient was a healthy 45-year-old woman attending our clinic with a diagnosis of a malerupted and semi-impacted right mandibular 3rd molar. She had been complaining of non-localized recurrent pain in the distal area of the right mandible for approximately 2 months. Swelling in the right submandibular area had caused asymmetry of the face. After visiting her local dentist, the patient had been referred to our hospital for an orthopantomogram (OPG) (Fig. 1) and then to a dental surgeon for extraction of the right mandibular 3rd molar.

A physical examination revealed that the right submandibular lymph node was enlarged to approximately $0.5 \mathrm{~cm}$ in diameter. It was slightly painful on palpation and was

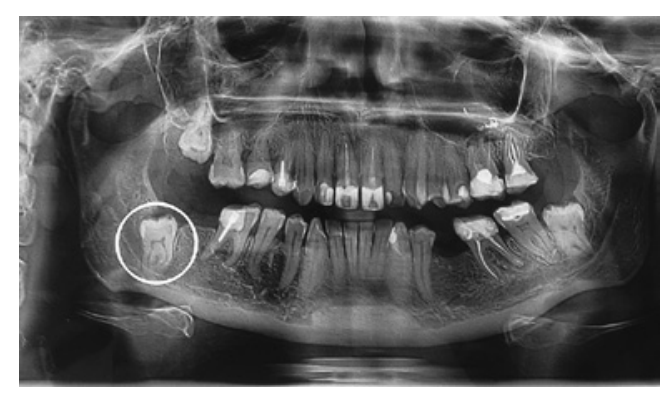

Fig. 1 Patient no.1: Dental panoramic radiograph revealed that crown of right 3rd molar (outlined) was half covered with bone visor from distal side; its medial tubercle was located superior to cortical plate of alveolar crest; molar roots were observed infiltrating mandibular canal; no signs of bone destruction were evident 


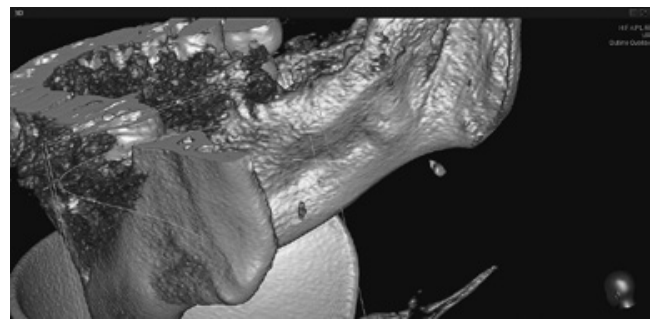

Fig. 2 Patient no.1: Fragments of 3D-CT scan of showed clearly visible sialolith when not superimposed on mandible

movable.

Intraoral examination revealed that the right mandibular 3rd molar was covered with pale-pink oral mucosa. Palpation of the retromolar trigone and transitory fold elicited no pain response. Therefore, removal of the right mandibular 3rd molar was not indicated. In contrast to the left one, palpation of the right sublingual fold caused discomfort. The right Wharton's duct was smooth and had an intermittent structure. Only poor secretion was observed from the right sublingual caruncle. Taken together, this led us to suspect dysfunction of the salivary gland, despite the absence of any sign of a sialolith on OPG.

Subsequently, US of the submandibular gland was performed together with 3D-CT of the mandible, with the latter revealing a sialolith (Fig. 2). The US allowed us to determine the size of the sialolith, its localization in Wharton's duct, and the isoechogenic structure of the glandular parenchyma.

The patient was then referred to maxillofacial surgery, where she successfully underwent intraoral surgical removal of the sialolith. At a follow-up examination at 7 days postoperatively, the patient presented with a complete resolution of symptoms. The prognosis in regard to sialolithiasis is favorable, due to the low recurrence rate r). $^{7}$.

\section{Case no.2}

This patient was a healthy 57-year-old woman who attended the university dental clinic with the chief complaint of pain radiat- ing to the right ear and swelling in the same submandibular area. These symptoms had arisen the previous day. The patient felt discomfort in the root of the tongue on the right when swallowing.

Anamnesis revealed submandibular lymphadenitis, which had been treated with partial success 6 months earlier. She mentioned that the right mandibular 1st and 2nd molars had been extracted after unsuccessful conservative treatment approximately 32 years earlier. She also told us that she had been given a diagnosis of a suppurated ameloblastoma of the right mandibular ramus at that time, and that this has been surgically treated with curettage and simultaneous extraction of the right 3rd mandibular molar. Since then, the patient had periodically felt the taste of pus, which resulted in frequent visits to her dentist.

Examination of the right submandibular area revealed a dense, non-elastic swelling $2 \mathrm{~cm}$ in diameter, which was mildly painful, slightly movable, and was not connected with the surrounding tissue. This swelling was covered in healthy epithelium. Intraoral palpation of the right sublingual fold caused discomfort, and no secretion was released from the right paramedian sublingual caruncle.

The patient had also undergone dental OPG 4 times within the previous 9 years, revealing multilocular radiolucent lesions of the right mandibular ramus corresponding to an ameloblastoma, and clearly visible radiopaque calculus, which was superimposed on the mandible, but undiagnosed.

The preliminary diagnosis was sialolithiasis in the right submandibular salivary glands, which was confirmed by 3D-CT (Fig. 3). High frequency ultrasound revealed a sialolith in the parenchyma of the glands.

Since ameloblastoma is a painless disor$\operatorname{der}^{3}$, it was determined that the sialolithiasis was responsible for the patient's reported discomfort over the years. The patient was referred to maxillofacial surgery for gland removal, but surgical intervention was postponed due to increased total bilirubin in her blood. Radical resection of the jaw ramus was 


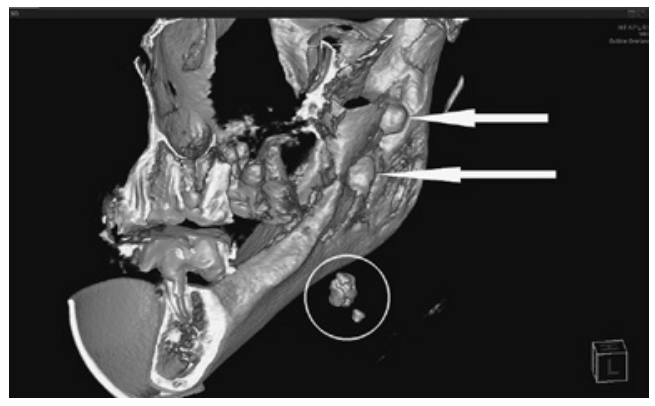

Fig. 3 Patient no.2: Fragment of 3D-CT scan showed sialolith (outlined) in right submandibular area; right mandibular ramus showed deformation (arrows) by neoplastic (benign) process

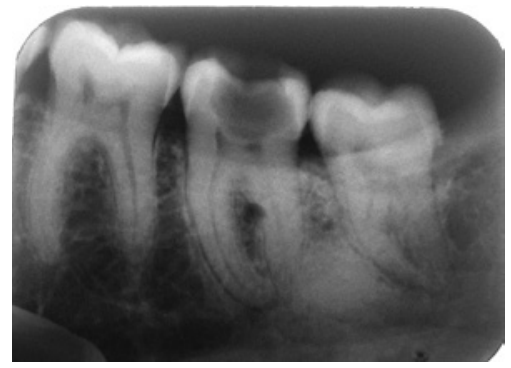

Fig. 4 Patient no.3: Periapical radiography of left mandibular molars showed focus of bone induration of approximately $5 \mathrm{~mm}$ in diameter with clear border, probably osteoma, localized close to apical third of distal root of left 2nd mandibular molar

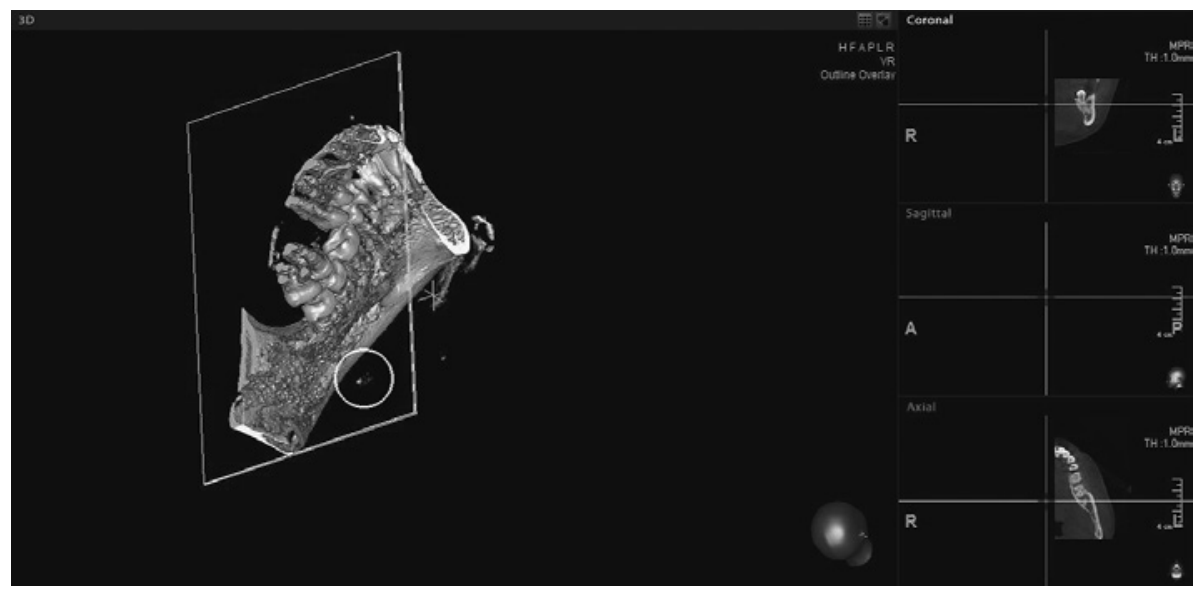

Fig. 5 Patient no.3: 3D-CT revealed sialolith in submandibular region

indicated for the ameloblastoma ${ }^{3)}$, but was postponed as the patient refused. At present, the patient's condition is satisfactory, and she is aware of a possible unfavorable prognosis.

\section{Case no.3}

This patient was a healthy 40-year-old woman who attended the university dental clinic for consultation on a radiographically detected osteoma adjacent to the distal root of the left mandibular 2nd molar (Fig. 4).

The patient complained of dull pain in the left submandibular area in the evenings and discomfort during swallowing. These symptoms had appeared 4 days earlier, along with an increase in toothache when biting. This toothache resolved, however, the next day, while pain in the submandibular area sharpened.

Extraoral examination revealed no significant swelling or lymphadenopathy. Intraoral examination revealed extensive cavitation in the left mandibular 2nd molar and an enlarged orifice of Wharton's duct on the left side. The duct itself was deformed, and bimanual palpation of the floor of the mouth 
caused pain but no saliva secretion. Consequently, sialolithiasis was suspected as the most likely cause.

High frequency ultrasound revealed a sialolith approximately $1.0 \mathrm{~mm}$ in diameter in the dilated left Wharton's duct. The glandular tissue was isoechogenic in structure. A diagnosis of sialolithiasis of the left submandibular salivary gland was confirmed by 3D-CT (Fig. 5).

An osteoma and endodontic problems with the left mandibular 2nd molar were the preferential initial clinical findings. However, a more thorough clinical examination suggested submandibular sialolithiasis.

Subsequently, endodontic treatment of the molar was carried out first. The sialolith, was treated conservatively as it was small. An osteoma is considered to be an inactive neoplasm, and only a single case of malignant transformation of an osteoid osteoma into low grade aggressive osteoblastoma has been reported in the literature ${ }^{9)}$. Therefore, it was decided to conduct regular follow-up of the neoplasm. To date, the patient has made no further complaints at subsequent follow-up examinations. As to the prognosis, according to the literature, the recurrence rate of sialolithiasis is approximately $8.9 \%{ }^{7)}$. The prognosis in regard to osteoma is in generally favorable.

\section{Discussion}

Despite being one of the most prevalent disorders of the submandibular salivary glands, the diagnosis of sialolithiasis can still be a challenge, especially in dentistry ${ }^{2}$. Cases of sialolithiasis, and methods for its visualization and removal, have frequently been reported $^{2,46}$. One feature of the present report is the combination of dental and nondental pathologies, with sialolithiasis of the submandibular salivary glands affecting the same sextant.

Case no.1 showed signs common to two different diseases: pericoronitis and submandibular sialolithiasis. The difficulty in diagnosing sialolithiasis of the submandibular sali- vary glands here was mostly due to the nonradiopacity of the sialolith. Fortunately, an appropriate and relatively timely diagnosis allowed for the surgical extraction of a malerupted and semi-impacted right mandibular 3rd molar, which had been initially indicated, and which is rather traumatic and can incur the risk of complications due to its interrelationship with the mandibular canal, to be abandoned (Fig. 1).

In case no.2, a previously diagnosed ameloblastoma, which was the preferential radiographic finding, rendered sialolithiasis imperceptible for at least 9 years, despite the radiopacity of the sialolith. Here, exacerbation of sialolithiasis was mistaken for lymphadenitis, again, despite the radiopacity of sialolith. This also entailed a loss of time for resection of the salivary gland.

In case no.3 sialolithiasis was accompanied by endodontic problems and a minor endosteal osteoma. Here, the sialolithiasis had to be treated taking into account the other condition. The sialolithiasis in this case was diagnosed in a timely manner, despite the dentists' attention initially being focused on odontopathological symptoms.

Diagnostic algorithms in dentistry are based on the odontogenic causes of the problem. This being the case, a chronic process, such as sialolithiasis in the submandibular salivary gland in the presence of affected teeth in the same quadrant, has almost no chance of being immediately identified by a dentist. In such a case, the diagnostic search can take years, as shown in case no.2 here. Only after exclusion of all other possible dental diagnoses, and probably only after treatment or extraction of teeth, will persistent clinical presentation be likely to lead to examination of the salivary glands and a 3D-CT study of the jaws, which is the only way that a sialolith, even one that was radiopaque, would be identified.

In all the present cases, 3D-CT was used as the method of choice for visualization of the sialoliths. Meanwhile, one study has reported that US is the first choice for such examination in order to screen for and exclude paren- 
chymal and intraductal morphological changes $^{1)}$. A differential diagnosis of sialadenitis includes the following causes, most of which are either unknown to or beyond the scope of expertise of the dentist in general practice: acute infection; inflammation; autoimmune dysfunction; granulomatosis; medication; neoplastic (benign and malignant) masses; endocrinal dysfunction; and metabolic disorders ${ }^{4}$. This means that, in certain cases, some pathological changes can only be identified by CT of soft tissues or sialendoscopic examination and a biopsy, often in consultation with a medical specialist.

\section{Conclusion}

The results of the present report on 3 clinical cases emphasize the necessity of the dentist also taking into account potential nondental causes of pain in the submandibular area together with submandibular lymphoadenopathies in arriving at a differential diagnosis. Despite new developments in imaging technologies and diagnostics, sialolithiasis continues to be misdiagnosed in clinical practice. The atypical clinical features of sialolithiasis of the submandibular salivary glands need to be further explored and their significance better understood.

\section{Acknowledgements}

We would like to thank Vitaliy Dubina for their technical assistance.

\section{Conflict of Interest}

The authors declare that they have no financial interests, direct or indirect, that exist or might be perceived to exist in connection with the contents of this paper.

Source of financial support or funding: Nil.

\section{References}

1) Andretta M, Tregnaghi A, Prosenikliev V, Staffieri A (2005) Current opinions in sialolithiasis diagnosis and treatment. Acta Otorhinolaryngol Ital 25:145-149.

2) Capaccio P, Marciante GA, Gaffuri M, Spadari F (2013) Submandibular swelling: Tooth or salivary stone? Indian J Dent Res 24:381-383.

3) Effiom OA, Ogundana OM, Akinshipo AO, Akintoye SO (2017) Ameloblastoma: current etiopathological concepts and management. Oral Diseases [serial online]. First published: 9 March 2017. doi: 10.1111/odi.12646.

4) Iro H, Zenk J (2014) Salivary gland diseases in children. GMS Curr Top Otorhinolaryngol Head Neck Surg 13: Doc06. doi: 10.3205/ cto000109.

5) Jager L, Menauer F, Holzknecht N, Scholz V, Grevers G, Reiser M (2000) Sialolithiasis: MR sialography of the submandibular duct - an alternative to conventional sialography and US? Radiology 216:665-671.

6) Kim JH, Aoki EM, Cortes ARG, Abdala-Júnior R, Asaumi J, Arita ES (2016) Comparison of the diagnostic performance of panoramic and occlusal radiographs in detecting submandibular sialoliths. Imaging Sci Dent 46:87-92. doi: 10.5624/isd.2016.46.2.87.

7) Lustmann J, Regev E, Melamed Y (1990) Sialolithiasis. A survey on 245 patients and a review of the literature. Int J Oral Maxillofac Surg 19:135-138. doi: 10.1016/S0901-5027 (05) 80127-4.

8) Marchal F, Dulguerov P (2003) Sialolithiasis management: the state of the art. Arch Otolaryngol Head Neck Surg 129:951-956. doi: 10.1001/archotol.129.9.951.

9) Mohammed I, Jannan NA, Elrmali A (2013) Osteoid osteoma associated with the teeth: unusual presentation. Int J Oral Maxillofac Surg 42:298-302.

10) Schrøder SA, Andersson M, Wohlfahrt J, Wagner N, Bardow A, Homøe P (2017) Incidence of sialolithiasis in Denmark: a nationwide population-based register study. Eur Arch Otorhinolaryngol 274:1975-1981. doi: $10.1007 /$ s00405-016-4437-z.

Correspondence:

Dr. Viktoriya Shynkevych

Department of Postgraduate Education for Dentists,

Higher State Educational Establishment of Ukraine

"Ukrainian Medical Stomatological

Academy", Poltava, Ukraine

E-mail: shinkevichvictoria@gmail.com 